\title{
Aktuelle Projekte und Leitlinien zum Umgang mit Multimedikation
}

Im Jahr 2005 beschrieben Cynthia Boyd und Kollegen die Problematik der Anwendung verschiedener fachbezogener Leitlinien für eine hypothetische 79-jährige Patientin mit Osteoporose, Arthritis großer Gelenke, Diabetes mellitus Typ 2, Hypertonie und chronisch obstruktiver Atemwegserkrankung (COPD) [1]. Die Einnahme der täglichen Medikation war mit 19 Medikamentenanwendungen zu 5 Tageszeiten verbunden, hinzu kamen zahlreiche komplexe nicht-pharmakologische Hinweise wie z. B. Gymnastik. Die kritische Analyse der fachbezogenen Leitlinien ergab, dass nur 4 von 9 LL Komorbiditäten adressierten und keine einzige von ihnen auch Patientenpräferenzen einschloss.

Im Kontext der Fragestellungen Multimorbidität und Multimedikation (Polypharmazie) sollten zunächst die Begriffe definiert werden. Während Multimorbidität ursprünglich das Vorliegen von mindestens zwei chronischen Erkrankungen beschrieb, wird nun zwischen Komorbidität (eine „dominierende“ Diagnose und Begleiterkrankungen) und Multimorbidität (paralleles und gleichberechtigtes Vorliegen mehrerer chronischer Erkrankungen) unterschieden [2]. Während aus der Perspektive z.B. eines Kardiologen eine Patientin an symptomatischer Herzinsuffizienz leidet, werden im Behandlungskontext Depression und Arthrose als Komorbidität betrachtet. Aus der Perspektive des Hausarztes und der Patientin schränken alle drei Erkrankungen ihre Mobilität ein und verstärken sich gegenseitig. Aus beiden Blickwinkeln heraus resultiert jedoch häufig eine Polypharmazie, die meist mit 5 oder mehr regelmäßig verordneten Medikamenten definiert wird [3]. Hinzu kommt, dass für die Multimedikation und Polypragmasie keine qualitativ hochwertige Evidenz für deren Nutzen vorliegt: multimorbide Patienten sind in klinischen Studien in der Regel ausgeschlossen, und epidemiologische Studien deuten alle darauf hin, dass in der Polypharmazie mehr Gefahren als Nutzen liegen [4]. Polypharmazie ist assoziiert mit zahlreichen Interaktionen, unerwünschten Ereignissen, geringerer Lebensqualität, Krankenhausaufnahmen und erhöhter Mortalität [5].
Aus diesem Dilemma resultiert eine zentrale Frage, die bei der Entwicklung von Leitlinien Berücksichtigung finden sollte: Welche Aspekte sind bei der Behandlung der chronischen Erkrankung X (z. B. Diabetes mellitus Typ 2) zu berücksichtigen, wenn Multimorbidität und Polypharmazie bereits vorliegen? Dahinter verbergen sich die eher pharmakologischen Fragestellungen: Welche Arzneimittel-Krankheits-Interaktionen können auftreten (z.B. Betablocker nach Herzinfarkt bei gleichzeitiger schwerer (OPD) und welche ArzneimittelInteraktionen können auftreten (z. B. orale Antikoagulation und Schmerztherapie mit NSAR) und wie beeinflussen komplexe Therapieregime die Adhärenz des Patienten [6]? Hinzu kommen die vorwiegend auf Multimorbidität und Alter bezogenen Fragen nach Evidenz einer Therapie in diesem individuellen Kontext und Präferenzen des Patienten; wann ist weniger mehr [7]. Gerade aus der Allgemeinmedizin kam national und international die Frage nach der Anwendbarkeit fachspezifischer Leitlinien für multimorbide Patienten mit vielen Medikamenten auf $[1,8]$.

In Deutschland wurde erstmals 2013 die Hausärztliche Leitlinie Multimedikation herausgegeben [9], die 2019 aktualisiert werden soll. In Ergänzung zu fachspezifischen Fragen wird auf in der $>$ Tab. 1 genannte Punkte hingewiesen, die zum Erfolg oder Misserfolg einer Pharmakotherapie beitragen können. Zu den hier aufgeführten Aspekten kommt noch eine Überprüfung auf Interaktionen hinzu, die von der eigenen Verordnungssoftware oder durch Apotheker unterstützt werden kann.

Wie so häufig im hohen Lebensalter und bei Multimorbidität ist auch die Evidenz für Ansätze zur „Optimierung“ der Polypharmazie recht dünn. Überwiegend komplexe, multiprofessionelle interventionelle Studien haben sich in unterschiedlichen Settings, d.h. ambulant/stationär/stationäre Altenpflege mit diesem Thema befasst. Das nüchterne Fazit lautet: Die Evidenz für den Nutzen im Hinblick auf „Medikationsqualität“, meist gemessen als der Medication Appropriateness Index (MAI), ist schwach. Dass Interventionen Patienten-bezogene Outcomes wie Hospi- talisierungen oder Lebensqualität verbessern, ist ebenfalls nicht sicher belegt [10]. Gray et al. kommen zu dem Schluss, dass komplexe pharmazeutische Interventionen zu einer signifikanten Reduktion von unerwünschten Arzneimittel-assoziierten Ereignissen führen, wobei methodische Qualität und Reporting Bias bemängelt werden, ebenso wie die Tatsache, dass die meisten Studien monozentrisch durchgeführt wurden und die Übertragbarkeit unklar sei [11].

In der PRIMUM-Studie wurde bei 505 Patienten in 72 hessischen Hausarztpraxen Cluster-randomisiert ein Medikationsreview durchgeführt [12]. Im Ergebnis wurden weder der MAI noch die Lebensqualität signifikant beeinflusst. Die EU-geförderte PRIMA-eDS Studie fand in 4 europäischen Ländern statt, darunter auch Deutschland [13]. In der Interventionsgruppe wurde den Hausärzten eine elektronische Verordnungsunterstützung zum Evidenz-basierten Absetzen häufig vorkommender Medikamente zur Verfügung gestellt. Hierzu waren umfangreiche Vorarbeiten erforderlich, d.h. systematische Reviews zur Evidenz z. B. von oralen Anti-

- Tab. 1 Praktische Empfehlungen der hausärztlichen Leitlinie Multimedikation (nach [9]).

einmal jährliche Bestandsaufnahme der gesamten Medikation, Anwendungsprobleme, Verdacht auf Nebenwirkungen, frei erhältliche Medikation, Adhärenz

Indikation der Therapie (noch) gegeben, Dosierung korrekt, Verträglichkeit für jedes Medikament prüfen

Patientenpräferenzen zu seiner Therapie erfragen und berücksichtigen

Weiterverordnung oder Absetzen für jedes Medikament überprüfen

Kommunikation mit dem Patienten zu seiner Medikation, Medikationsplan aktuell halten und aushändigen

ausreichendes Monitoring der Wirksamkeit und Verträglichkeit der Medikation (z. B. einmal jährliche Kontrolle der Nierenfunktion), aber auch auf Symptome achten

bei Facharztbesuchen, Krankenhausaufenthalt: Medikationsplan erneuern und prüfen 
diabetika, Antihypertensiva, oralen Antikoagulantien, bei betagten Patienten ([14], s.u.).

Bei den bekannten Risiken der (insbesondere unkontrollierten) Polypharmazie betrifft eine wesentliche Frage das Absetzen von möglicherweise unverträglichen Medikamenten, für deren weitere Anwendung keine gesicherte Evidenz (mehr) besteht. Der Vorgang des systematischen Absetzens von Medikamenten wird international „De-prescribing“ genannt. Scott et al. beschreiben detailliert die Barrieren (z.B. Ängste von Ärzten und Patienten, eine möglicherweise hilfreiche Therapie zu unterlassen) und das praktische Vorgehen beim Absetzen von Medikamenten [15]. Hierbei spielen neben der Evidenz die Arzt-Patientenkommunikation sowie das Einbeziehen der Angehörigen eine entscheidende Rolle. So wie es für Akutmedikation (z. B. Antibiotika) die Rationale für die Beendigung einer Therapie gibt, sollte die gesamte Pharmakotherapie unter dem Gesichtspunkt eines Prozesses betrachtet werden, an dessen Ende auch das Absetzen stehen kann. Mittlerweile existieren randomisierte, kontrollierte Studien zum De-prescribing und auch Leitlinien, beispielsweise für orale Antidiabetika [16]. Das Design und die Durchführung von De-prescribing-Studien stellt eine Herausforderung dar; zahlreiche Projekte werden national und international mit öffentlichen Mitteln gefördert.

\section{Interessenkonflikt}

Die Autorin gibt an, dass kein Interessenkonflikt besteht.

\section{Autorinnen/Autoren}

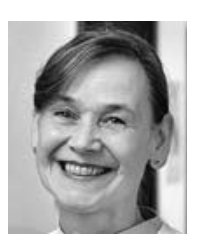

Petra A. Thürmann

Prof. Dr. med., Lehrstuhl für Klinische Pharmakologie, Universität Witten/Herdecke, Helios Universitätsklinikum Wuppertal
Korrespondenzadresse

Prof. Dr. med. Petra A. Thürmann

Lehrstuhl für Klinische Pharmakologie Universität Witten/Herdecke

Helios Universitätsklinikum Wuppertal

Heusnerstraße 40

42283 Wuppertal

petra.thuermann@helios-gesundheit.de

\section{Literatur}

[1] Boyd CM, Darer J, Boult C et al. Clinical practice guidelines and quality of care for older patients with multiple comorbid diseases: implications for pay for performance. JAMA 2005; 294: 716-724

[2] Nicholson K, Makovski TT, Griffith LE et al. Multimorbidity and comorbidity revisited: refining the concepts for international health research. J Clin Epidemiol 2019; 105: 142146

[3] Gnjidic D, Hilmer SN, Blyth FM et al. Polypharmacy cutoff and outcomes: five or more medicines were used to identify communitydwelling older men at risk of different adverse outcomes. J Clin Epidemiol 2012; 65: 989-995

[4] Nationale Akademie der Wissenschaften Leopoldina; acatech - Deutsche Akademie der Technikwissenschaften; Union der deutschen Akademien der Wissenschaften. Medizinische Versorgung im Alter - Welche Evidenz brauchen wir? Halle (Saale) September 2015. Im Internet: https://www.akademienunion.de/fileadmin/redaktion/user_ upload/Publikationen/Stellungnahmen/ 3Akad_Stellungnahme_Evidenzbasierung_ 2015_web.pdf; Stand: 24.09.2019

[5] Wastesson JW, Morin L, Tan ECK et al. An update on the clinical consequences of polypharmacy in odler adults: a narrative review. Exp Opinion Drug Saf 2018; 17: 1185-1196

[6] Rottlaender D, Scherner M, Schneider T et al. Multimedikation, Compliance und Zusatzmedikation bei Patienten mit kardiovaskulären Erkrankungen. Dtsch Med Wochenschr 2007; 132: 139-144

[7] American Geriatrics Society Choosing Wisely Workgroup. American Geriatrics Society identifies five things that healthcare pro- viders and patients should question. I Am Geriatr Soc 2013; 61: 622-631

[8] Cox L, Kloseck M, Crilly R et al. Underrepresentation of individuals 80 years of age and older in chronic disease clinical practics guidelines. Can Fam Physician 2011; 57: e263-e269

[9] Bergert FW, Braun M, Ehrenthal $\mathrm{K}$ et al. Hausärztliche Leitlinie Multimedikation. Version 1.09 vom 16.4.2014. AWMF Registernummer 053 - 043. Im Internet: https:// www.awmf.org/uploads/tx_szleitlinien/053043I_S2e_Multimedikation_2014-05abgelaufen.pdf; Stand: 09.09.2019

[10] Rankin A, Cadogan CA, Patterson SM et al. Interventions to improve the appropriate use of polypharmacy for older people. Cochrane Database Syst Rev 2018; (9): CD008165

[11] Gray SL, Hart LA, Perera S et al. Meta-analysis of interventions to reduce adverse drug reactions in older adults. J Am Geriatr Soc 2018; 66: $282-288$

[12] Muth C, Uhlmann L, Haefeli WE et al. Effectiveness of a complex intervention on prioritising multimedication in multimorbidity (PRIMUM) in primary care: results of a pragmatic cluster-randomised controlled trial. BMJ Open 2018; 8: e017740

[13] Sönnichsen A, Trampisch US, Rieckert A et al. Polypharmacy in chronic diseases-Reduction of Inappropriate Medication and Adverse drug events in older populations by electronic Decision Support (PRIMA-eDS): study protocol for a randomized controlled trial. Trials 2016; 17: 57

[14] Martinez YV, Renom-Guiteras A, Reeves D et al. A set of systematic reviews to help reduce inappropriate prescribing to older people: study protocol. BMC Geriatr 2017; 17 (Suppl. 1): 231

[15] Scott IA, Hilmer SN, Reeve E et al. Reducing inappropriate polypharmacy: the process of deprescribing. JAMA Intern Med 2015; 175: 827-834

[16] Farrell B, Black C, Thompson W et al. Deprescribing antihyperglycemic agents in older persons: Evidence-based clinical practice guideline. Can Fam Physician 2017; 63 : 832-843 